\title{
Influence of Atorvastatin/Probucol Combination on Blood Lipid and Serum C-Reactive Protein Levels in Patients with Cerebral Infarction
}

\author{
Tianshu Wang, Chun Li, Wenjing Deng, Ruina Niu, Ran Du and Junfang Teng* \\ Department of Neurology, the First Affiliated Hospital of Zhengzhou University, Zhengzhou, China
}

*For correspondence: Email: tjf05656@163.com

Received: 6 June 2015

Revised accepted: 3 November 2015

\begin{abstract}
Purpose: To explore effects of atorvastatin in combination with probucol on blood lipid and serum Creactive protein of patients with cerebral infarction.

Methods: In total, 120 patients with confirmed cerebral infarction were randomly divided into test and control groups ( $n=60$ each). Control group was given atorvastatin $20 \mathrm{mg}$, once every night, while test group received $0.5 \mathrm{~g}$ probucol twice daily plus atorvastatin $20 \mathrm{mg}$ nightly. The treatment for both groups lasted for six months. Changes in carotid intima media thickness (CIMT), plaque area, vulnerability, high-sensitivity C-reactive protein level (hs-CRP) and low density lipoprotein (LDL) were observed before and after treatment and compared between groups. In 6-month follow up, incidence of newly occurred or reoccurred cerebral ischemic stroke were observed and compared between groups. Occurrence of severe untoward reaction was also noted.

Results: In test group, LDL level significantly decreased after treatment, $(p<0.01)$, and when compared to control group ( $p<0.05)$. CIMT, plaque area and number of vulnerable plaque after treatment were significantly different compared to the values prior to treatment and control group ( $p<$ 0.05). Incidence of cerebral ischemic stroke was similarly lower for the test group than control after treatment $(p<0.05)$. Both groups did not develop any severe untoward reaction.

Conclusion: Atorvastatin, in combination with probucol, can be used to regulate blood lipid, lower LDL level, reduce number of vulnerable plaque, stabilize and reverse plaque, and lower risk of cerebral ischemic stroke of carotid atherosclerostic plaque. This work provides a theoretical basis and intervention measure for preventing ischemic stroke.
\end{abstract}

Keywords: Probucol, Atorvastatin, C-reactive protein, Cerebral infarction, Carotid plaque, Blood lipid

Tropical Journal of Pharmaceutical Research is indexed by Science Citation Index (SciSearch), Scopus, International Pharmaceutical Abstract, Chemical Abstracts, Embase, Index Copernicus, EBSCO, African Index Medicus, JournalSeek, Journal Citation Reports/Science Edition, Directory of Open Access Journals (DOAJ), African Journal Online, Bioline International, Open-J-Gate and Pharmacy Abstracts

\section{INTRODUCTION}

Cerebral infarction with high incidence rate, mortality rate, recurrence rate and disability rate is more likely to happen in middle aged and elderly people; it has been the primary cause for long-term disability of middle aged and elderly people worldwide, and also the second cause leading to death in China [1].
Carotid atherosclerostic plaque is considered as the primary cause of the occurrence of cerebral infarction [2]. Detecting the stability of carotid atherosclerostic plaque as soon as possible and using drugs that are beneficial for the stability of plaque are of great importance to prevent the occurrence of cerebral infarction clinically. In 2014, Hao et al [3] have proved that atorvastatin combined with probucol does not only improve adiponectin level of cerebral infraction patients 
but also lower high-sensitivity C-reactive protein (hs-CRP) level, which is beneficial to stabilize atherosclerotic plaque and control the occurrence of cerebral infarction. Zhang [4] pointed out that probucol combined with statin was effective in resisting inflammation and improving the level of adiponectin in the treatment of acute myocardial infarction (AMI), thereby protecting angiocarpy. Based on experiments, Gao et al [5] found that, statins could effectively control and lower CRP level and blood lipid, reduce inflammation and eliminate carotid atherosclerostic plaque; and atorvastatin could reduce carotid intima media thickness (CIMT) while being used in secondary prevention.

This study used atorvastatin combined with probucol to treat cerebral infarction patients and analyzed the influence of atorvastatin combined with probucol on blood lipid and serum Creactive protein of patients with cerebral infarction by performing ultrasonic blood vessel detection and $A D$ detection to.

\section{EXPERIMENTAL}

\section{Research objects}

A total of 120 patients who were verified to have carotid atherosclerostic plaque in First Affiliated Hospital of Zhengzhou University, Zhengzhou, China from May 2010 to April 2011 were selected. Of them, 50 cases had transient ischemic attack/minor stroke, based on National Institute of Health stroke scale (NIHSS) score criteria of $<4$ points. All cases conformed to the diagnostic criteria of carotid artery atherosclerosis formulated by WHO [6]. Clinical symptoms, sign and diagnostic results of computed tomography (CT) or magnetic resonance imaging (MRI) of cases of transient ischemic attack/ minor stroke all conformed to the diagnostic criteria stipulated by the 4th Cerebrovascular Disease Conference held by Chinese Medical Association [7]. All participants signed inform consent and this study has been approved by the ethics committee of the First Affiliated Hospital of Zhengzhou University, Zhengzhou, China.

\section{Inclusion criteria of cerebral infarction}

Patients who were confirmed by $\mathrm{CT}$ and $\mathrm{MRI}$ to conform to the guidelines of 4th National Conference on the Diagnosis of Cerebrovascular Disease were included in the study. Also, patients who were confirmed to have carotid arteriosclerosis plaque by vascular ultrasound and who had CIMT on both sides or one side $\geq$ to $1.0 \mathrm{~mm}$ were also included.

\section{Exclusion criteria}

Patients who had a large area of cerebral infarction, IMT less than $1.0 \mathrm{~mm}$ or diseases such as rheumatic heart disease, atrial fibrillation, obvious hepatic failure and liver and kidney diseases were all excluded from the study.

\section{Grouping}

The recruited patients were randomly divided into test group and treatment group, 60 in each group. The test group comprised of 35 males and 25 females, ranging in age from $45 \sim 76$ years (mean, $59.3 \pm 7.2$ years), while control group had 38 males and 22 females, ranging in age from 44 to 78 years (mean, $58.6 \pm 7.9$ years). Differences in demographic characteristics between the two groups with regard to age, gender, weight index, smoking condition, drinking condition, hypertension and diabetes were not statistically significant $(p>0.05)$.

\section{Therapeutic method}

Control group was given diet and living style improvement therapy, $20 \mathrm{mg}$ atorvastatin calcium tablets Lipitor®, Huirui Pharmaceutical Co., Ltd, China) once nightly, as well as treatment for controlling blood pressure, blood glucose, smoking cessation and antiplatelet. For the treatment group, patients were given $0.5 \mathrm{~g}$ probucol (twice each day) plus atorvastatin (20 $\mathrm{mg}$, once nightly). Six $\mathrm{ml}$ venous blood was extracted from patients before treatment and also 6 months after treatment to assess low density lipoprotein (LDL), blood lipid, high-sensitivity Creactive protein (hs-CRP), blood routine, liver renal function and creatine kinase. In addition, the blood vessels of the neck were examined by color Doppler ultrasound. LDL detection kit was used enzyme colorimetry (Ningbo Meikang Biotech Science and Technology Co., Ltd., China) with the aid of BecKman DXC-800 automatic analyzer while hs-CRP was determined using rate scatter turbid assay and brain natriuretic peptide (BNP) analyzer (Siemens Medical Diagnosis Product Co., Ltd, Shanghai, China).

In addition, Philips IU22 color ultrasonography was used to detect bilateral common carotid artery, carotid bulb and extracranial internal carotid artery. Patients were asked to fully expose their lateral neck by taking supine position with the head towards tilted to the 
unexamined side. L9-3 two-dimensional linear probe was applied to perform whole-process monitoring on carotid artery, e.g., observe condition of intima, size, form and echo characteristics of plaque and degree of stenosis of lumen. If the site that was $1.5 \mathrm{~cm}$ away from bifurcation of common carotid artery had atherosclerotic plaque, then vertical distance between intima and interface between media membrane and outer membrane was measured. The mean of the two was taken as the final result.

\section{Statistical analysis}

All the data were processed by SPSS 17.0. Data were expressed as mean \pm standard deviation (SD). Comparison between two groups was performed by t test, and measurement data was compared by chi-square test. Difference was considered statistically significant if $p<0.05$.

\section{RESULTS}

\section{Comparison of blood fat level of two groups before and after treatment}

Blood fat level in both groups had no difference before treatment. In test group, total cholesterol (TC), low-density lipoprotein cholesterol (LDL-C) and triglyceride level obviously decreased $(p<$ 0.01 ), but high density lipoprotein-cholesterol (HDL-C) had no obvious decline $(p>0.05)$; in control group, TC, LDL and TG level showed a significant decrease $(p<0.01)$, but HDL level rose $(p<0.05)$.
After treatment, test group displayed lower TC and LDL-C levels than control group $(p<0.05)$; TG level was not significantly different between the two groups $(p>0.05)$; control group exhibited increased HDL-C, but test group showed no remarkable increase of HDL-C $(p>0.05)$. Details are shown in Table 1.

\section{Comparison of hs-CRP level of two groups before and after treatment}

hs-CRP level in two groups was not significantly different before treatment $(p>0.05)$. After treatment, hs-CRP level significantly decreased in both groups $(p<0.01)$; and a remarkable difference was also found between test group and control group $(p<0.05)$. Details are shown in Table 2.

\section{Comparison of CIMT and plaque area}

CIMT and plaque area decreased in both groups after treatment and there was remarkable difference within group $(p<0.05)$, and the difference between two groups was also statistically significant $(p<0.05)$, as shown in Table 3.

\section{Comparison of reduced number of vulnerable plaque before and after treatment}

Vulnerable plaque reduced in both groups after treatment and there was a remarkable difference within group $(p<0.05)$, and the comparison between two groups after treatment also showed a significant difference $(p<0.05)$ (Table 4).

Table 1: Comparison of blood lipid level of the two groups before and after treatment (mean $\pm \mathrm{SD}, \mathrm{mmol} / \mathrm{L}$ )

\begin{tabular}{lcccc}
\hline Item & \multicolumn{2}{c}{ Test group } & \multicolumn{2}{c}{ Control group } \\
\cline { 2 - 5 } & Before treatment & After treatment & Before treatment & After treatment \\
\hline TC & $5.52 \pm 1.12$ & $4.30 \pm 1.35^{\#} \Delta$ & $5.13 \pm 1.37$ & $3.10 \pm 0.95^{\#}$ \\
TG & $2.24 \pm 0.75$ & $1.51 \pm 0.35^{\#}$ & $2.32 \pm 1.12$ & $1.59 \pm 0.75^{\#}$ \\
LDL-C & $3.66 \pm 0.65$ & $2.14 \pm 0.65^{\#} \Delta$ & $3.75 \pm 0.51$ & $2.74 \pm 0.42^{\#}$ \\
HDL-C & $1.27 \pm 0.31$ & $1.07 \pm 0.28^{*}$ & $1.28 \pm 0.26$ & $1.55 \pm 0.31^{\#}$ \\
\hline
\end{tabular}

$\# p<0.01,{ }^{*} p>0.05$ compared to pre-treatment levels; for comparison of two groups after treatment, $\Delta$ indicates $p<0.05$

Table 2: Comparison of hs-CRP levels of the two groups before and after treatment (mean $\pm \mathrm{SD}, \mathrm{mg} / \mathrm{L})$

\begin{tabular}{|c|c|c|c|c|}
\hline \multirow[t]{2}{*}{ Item } & \multicolumn{2}{|c|}{ Test qroup } & \multicolumn{2}{|c|}{ Control group } \\
\hline & Before treatment & After treatment & Before treatment & After treatment \\
\hline hs-CRP & $8.03 \pm 1.12$ & $3.10 \pm 1.3^{\# \star}$ & $8.22 \pm 1.37$ & $4.56 \pm 1.29^{\#}$ \\
\hline
\end{tabular}


Table 3: Comparison of CIMT $(\mathrm{mm})$ and plaque area $\left(\mathrm{mm}^{2}\right)$ of two groups before and after treatment (mean \pm SD)

\begin{tabular}{|c|c|c|c|c|}
\hline \multirow{2}{*}{$\begin{array}{l}\text { Group } \\
(n=60)\end{array}$} & \multicolumn{2}{|c|}{ CIMT (mm) } & \multicolumn{2}{|c|}{ Plaque area $\left(\mathrm{mm}^{2}\right)$} \\
\hline & Before treatment & After treatment & $\begin{array}{c}\text { Before } \\
\text { treatment }\end{array}$ & After treatment \\
\hline Test group & $1.07 \pm 0.37$ & $0.92 \pm 0.24^{\#^{+}}$ & $0.81 \pm 0.55$ & $0.52 \pm 0.32^{\#}$ \\
\hline Control group & $1.05 \pm 0.39$ & $0.98 \pm 0.26^{\#}$ & $0.80 \pm 0.46$ & $0.68 \pm 0.54^{\#}$ \\
\hline
\end{tabular}

$P>0.05$ for comparison of two groups before treatment; ${ }^{*} p<0.05$ for comparison within group before and after treatment; ${ }^{*} p<0.05$ for comparison between two groups post-treatment

Table 4: Comparison of reduced number (rate) of vulnerable plaque of two groups before and after treatment $\left(x^{2}\right)$

\begin{tabular}{ccccc}
\hline Group & \multicolumn{2}{c}{ Vulnerable plaque } & \multicolumn{2}{c}{ Stable plaque } \\
\cline { 2 - 4 }$(\mathrm{n}=60)$ & Before treatment & After treatment & Before treatment & After treatment \\
\hline Test group & $31(51.6)$ & $21(35)^{\#}$ & $29(48.4)$ & $39(65)$ \\
Control group & $30(50.0)$ & $26(43.3)^{\#}$ & $30(50.0)$ & $34(56.7)$ \\
\hline
\end{tabular}

$P>0.05$ for comparison of the two groups before treatment; $\# p<0.05$ for comparison before and after treatment within the groups; * $p<0.05$ for comparison between two groups

\section{Untoward reaction}

In the treatment group, 3 cases had discomfort in upper abdomen in the 7th day after treatment, but were relieved after taking drugs. In control group, one case developed tolerable headache and relieved several days later. Liver function, renal function, blood routine and creatine kinase in both groups were within normal range before and after treatment.

\section{DISCUSSION}

Pathophysiologic process of cerebral infarction can be understood as local avascular necrosis of brain tissue occurring to/in patients with atherosclerosis. Brain tissue is especially sensitive to ischemia and oxygen deficit. Once the blood supply stops for 4 to $6 \mathrm{~min}$, unrecoverable injury is likely to occur. Thus, atherosclerosis is thought to be the basis of cerebral thrombosis, and also the most common factor and high risk factor of cerebral infarction occurrence [8].

Stability of carotid atherosclerostic plaque is considered to be closely correlated to the occurrence of cerebral infarction [9]; therefore, treatment of atherosclerotic lesion and stable plaque focuses on lipid regulation, oxidation resistance, anti-inflammation, platelet resistance and improvement of endothelial function [10]. In atherosclerotic lesion, lipid core and fibrous cap constitute the mature plaque. Based on data from vascular ultrasound, atherosclerotic plaque can be divided into low echo plaque and high echo plaque. High echo plaque, which is featured by a thick fibrous cap along with small and strong lipid necrotic core, is not easy to break, while low echo plaque featured by thin fibrous cap and big lipid necrotic core is easy to break. Instability of atherosclerosis is the leading cause and also the independent predictive factor for cerebral infarction. Cerebral infarction is based on atherosclerosis which is a chronic inflammatory reaction that occurs following blood vessel impairment. Several inflammatory cells and cytokines are involved in the occurrence and development of atherosclerosis [11].

Dyslipidemia is one of the most important risk factors of atherosclerosis; LDL-C can result in atherosclerosis, while HDL-C can resist it. A previous research [12] demonstrates that patient with dyslipidemia presents remarkably increased LDL-C and HDL-C. It has been found that inflammatory reaction plays an important role in the instability and disruption of atherosclerotic plaque. CRP, a sensitive index for detecting inflammatory reaction, correlates positively with severity of atherosclerosis, and it can be taken as the risk predictor of cardiovascular and cerebrovascular events that occur in patients with atherosclerosis [13].

Atorvastatin, a reductase inhibitor for hydroxymethyl-glutaryl-CoA (HMG-CoA), is a ratelimiting enzyme [14]. It can block endogenous cholesterol synthesis by inhibiting the reductase of HMG-CoA. Atorvastatin is able to stabilize and reduce plaque as it can increase the amount of LDL receptors on the surface of cell by induction, thus promoting catabiosis of LDL mediated by receptor. Probucol, an antioxidant certified by American Food and Drug Administration, plays the role of resisting oxidation, atherosclerosis and restenosis induced by angioplasty. To be specific, it can lower the level of ox-LDL, prevent or delay the occurrence and development of 
atherosclerosis and reduce the occurrence of myocardial infarction and stroke through multiple pathways [15].

The results of this study demonstrate that TC, TG, LDL-C and CRP significantly decreased after treatment in both groups, but the test group showed much lower values than control group after treatment; HDL-C of both groups increased significantly after treatment, but the test group exhibited higher values; IBS for AD of low echo plaque significantly improved in both groups, and again, the test group demonstrated greater improvement.

\section{Limitation of the study}

The sample size in this study was small. A larger sample size should be used in future studies.

\section{CONCLUSION}

Probucol in combination with atorvastatin is more effective in lowering TC, LDL-C and CRP level than atorvastatin. It regulates blood fat, resist oxidation and inflammation, improve vascular endothelial function, stabilize plaque, reverse plaque, and effectively restrain the occurrence and development of atherosclerotic lesion, Furthermore, The role of the combined therapy in preventing ischemic stroke significant. However, further studies are required to ascertain the full significance of this therapeutic strategy.

\section{ACKNOWLEDGEMENT}

The authors would like to extend their sincere gratitude to all those who variously helped during this study.

\section{REFERENCES}

1. Duan CM, Zou SF. Progress in Research of the Risk Factor of Cerebral Infraction. Chin J Pract Nerv Dis 2012; 15(3): 93-95.

2. Wang L, Sui XF. Correlation of Acoustic Feature of Atherosclerotic Plaque Detected by Ultrasound and
Occurrence of Cerebral Infarction. Anhui Med J 2014; 35(6): 831-832.

3. Hao YG, Chen WW, Zhao $Y, L i N$. Influence of Atorvastatin Combined with Probucol on Adiponectin and hs-CRP of Acute Cerebral Infarction Patients. Chin Pract Med 2014; 9(2): 153-154.

4. Zhang YJ. Effect of Probucol in Combination with Atorvastatin on Adiponectin and High Sensitive $C$ Reactive Protein in Patients with Acute Myocardial Infarction. Chin J Mod Drug Appl 2012; 6(6): 9-10.

5. Gao RL, Yao WH, Li BK. Influence of Atorvastatin on Carotid Atheromatous Plaque, Blood Lipid and Serum C-reactive Protein Level. Chin Foreign Women Health 2011; 19(7): 229.

6. Chen HZ. Internal Medicine. Beijing: People's Medical Publishing House 1989; 723-721.

7. Wu X. Summary of the 4th Cerebrovascular Disease Conference. Stroke Nerv Dis 1997; 4(2): 105-109.

8. Yang L. Influence of Atorvastatin on Blood Lipid and High Sensitive $C$ Reactive Protein. Chin J Pract Nerv Dis 2014; 17(17): 113-114.

9. Kerwin W, Hooker A, Spilker $M$, et al. Quantitative Magnetic Resonance Imaging Analysis of Neovasculature Volume Incarotid Atherosclerotic Plaque. Circulation 2003; 107(6): 851-856.

10. Zhao WJ, An ZP, Wang JH, Ning XJ. Relationships between Treatment at Acute Stage and the Prognosis in Atherothrombosis Ischemic Stroke Patients. Shandong Med J 2011; 51(9): 13-15.

11. Yamashita T, Hirata K. Anti-inflammatory and Immune Modulation Therapy against at Herosderosderosis. Nippon Rinsho 2011; 69(1): 138-172.

12. Koba S, Hirano T. Dyslipidemia and Atherosderosis. Nippon Rinsho 2011; 69(1): 138-143.

13. Wensley F, Gao P, Burgess $S$, et al. Association between $C$ Reactive Protein and Coronary Heart Diserse: Mendelian Randomization Andydsis based on Individual Participant Data. BMJ 2011; 342(548): 1-8.

14. Yin $L Y$, Zhang JP, Guo HF. Clinical Research on the Influence of Probucol Combined with Atorvastatin on Carotid Atherosclerostic Plaque. Capital Med 2010; 17(9): 53-54.

15. Qiao HG. Clinical Analysis of Atorvastatin Combined with Probucol Treating Instability of Carotid Atherosclerostic Plaque. Shanxi Med J 2014; 43(4):412-413. 